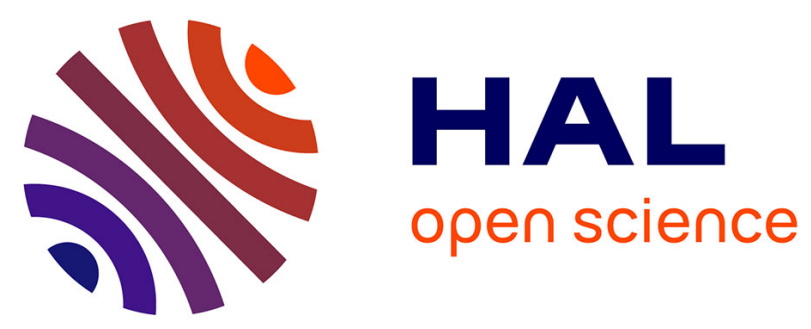

\title{
Pour une approche du paysage sonore par analogie au visuel - si le son donne à voir, le champ de vision donne-t-il à entendre?
}

Thomas Leduc, Philippe Woloszyn

\section{- To cite this version:}

Thomas Leduc, Philippe Woloszyn. Pour une approche du paysage sonore par analogie au visuel - si le son donne à voir, le champ de vision donne-t-il à entendre?. Claire Guiu; Guillaume Faburel; MarieMadeleine Mervant-Roux; Henry Torgue; Philippe Woloszyn. Soundspaces - Espaces, expériences et politiques du sonore, Presses Universitaires de Rennes, pp.241-250, 2015, 978-2-7535-3460-5. hal01347179

\author{
HAL Id: hal-01347179 \\ https://hal.science/hal-01347179
}

Submitted on 20 Jul 2016

HAL is a multi-disciplinary open access archive for the deposit and dissemination of scientific research documents, whether they are published or not. The documents may come from teaching and research institutions in France or abroad, or from public or private research centers.
L'archive ouverte pluridisciplinaire HAL, est destinée au dépôt et à la diffusion de documents scientifiques de niveau recherche, publiés ou non, émanant des établissements d'enseignement et de recherche français ou étrangers, des laboratoires publics ou privés. 


\title{
2.3. Pour une approche du paysage sonore par analogie au visuel - si le son donne à voir, le champ de vision donne-t-il à entendre ?
}

\author{
Thomas Leduc* et Philippe Woloszyn** \\ * LUNAM Université, CNRS, CERMA UMR 1563, École nationale supérieure d'architecture de Nantes, Nantes, \\ France \\ ** ESO - UMR 6590, CNRS/Université Rennes 2, Laboratoire Espaces et Sociétés Rennes, Université de Haute \\ Bretagne, Maison de la Recherche en Sciences Sociales, Rennes, France
}

\section{Introduction : conception allocentrée versus conception égocentrée de l'espace sonore}

Il s'agit ici d'exploiter des travaux liés à la quantification et la qualification du bassin de vision d'un observateur immergé en environnement urbain pour produire une cartographie sonore approchée de l'espace urbain en jeu. D'un point de vue méthodologique, nous considérons que la complexité du déroulé de façades immédiatement environnant est étroitement corrélée à la capacité de diffusion sonore des façades visibles. Les enjeux à l'œuvre sont :

- le diagnostic. L'idée est alors d'identifier les spécificités locales propre à un territoire urbain et ses éventuelles vulnérabilités ;

- la compréhension des phénomènes, afin d'identifier les causes plus spécifiquement liées au mode d'aménagement de l'espace construit ;

- l'anticipation et la prévention de nuisances extrêmes liées à des événements exceptionnels (et, idéalement, la conception de formes permettant de les réguler).

L'objectif n'est pas de produire une nouvelle « carte du bruit » en milieu urbain. Ce type de cartographie thématique correspond généralement à une représentation choroplèthe ${ }^{1}$ où la grandeur représentée est un indice énergétique tel qu'un niveau de bruit équivalent. Ces cartographies sonores reposent sur un recensement et une spatialisation des sources sonores réelles ou apparentes (telles celles produites par les principaux axes de circulation motorisée). Elles correspondent à une estimation de nuisance sonore $a$ priori, abstraction dé-corrélée de la notion même d'usage (au sens où l'individu « récepteur » n'est ni modélisé, ni réellement intégré). C'est une approche que nous pourrions qualifier de «sources-centrée » ou d'allocentrée ${ }^{2}$.

Notre intention est ici radicalement différente. Nous cherchons ainsi à estimer un potentiel de "diffusivité $»^{3}$ en tout point de l'espace ouvert urbain tel que perçu par un piéton en situation. Cette estimation est exclusivement morpho-dépendante, au sens où, pour son calcul, nous ne prenons en compte que la morphologie immédiate des lieux. La première originalité de notre approche est qu'elle est ainsi non pas « sources-centrée » mais bien « récepteur-centrée », anthropo-centrée, égocentrée.

L'autre originalité de notre approche tient au mode de calcul lui-même. Nous n'avons pas retenu les méthodes utilisées classiquement en acoustique telles que la méthode des radiosités, ou sa dérivée la

\footnotetext{
${ }^{1}$ Une carte est dite choroplèthe lorsqu'elle représente une grandeur (résultant d'une mesure statistique par exemple) par un dégradé de couleurs ou un motif. Une telle représentation permet de mettre en évidence des disparités de valeurs de cette même grandeur entre diverses régions de la carte.

${ }^{2}$ En psychologie, l'allocentrisme désigne le caractère d'un sujet centré ou orienté vers autrui et non vers luimême (comme le serait un sujet égocentré). Il ne doit pas être confondu avec l'orientation morale que constitue l'altruisme. Par extension, nous assimilons l'approche «sources-centrée » (qui est intrinsèquement exclusivement dépendante d'externalités à l'individu « récepteur ») à une orientation allocentrée.

${ }^{3}$ Le potentiel de diffusivité caractérise la capacité de l'espace environnant un émetteur acoustique à créer un champ diffus, caractérisé par un niveau sonore égal en tout point.
} 
méthode des sources-images. En effet, dans une telle approche, la source sonore (ou son «fantôme » issu de la première réflexion) rayonne et l'environnement bâti réagit par réflexion, absorption, etc. La méthode que nous avons retenue est d'inspiration « visibility studies». Elle consiste en une évaluation, en tout point d'intérêt de l'environnement d'étude, du bassin de visibilité correspondant. Plus précisément, pour des raisons d'efficacité, de robustesse et de facilité de mise en œuvre, nous avons opté pour la méthode des champs d'isovists ${ }^{4}$ de (Benedikt, 1979).

Après un exposé de la méthode que nous avons retenue, nous présentons un cas d'application pour un terrain nantais et discutons les résultats obtenus. Nous concluons cette présentation en insistant sur la nécessité de mettre en place un dispositif de validation in situ et d'intégrer, dans une approche plus systémique, le point de vue égocentré à l'approche allocentrée classique.

\section{Méthodologie}

\section{Modélisation de l'espace urbain et potentialité des indicateurs morphométriques}

En milieu urbain, l'acoustique dépend fortement de la configuration et de la distribution de l'espace bâti. Se focaliser sur la morphologie de l'environnement construit constitue une étape indispensable à la bonne connaissance de la configuration sonore des lieux.

Néanmoins, pour satisfaire une exigence de disponibilité de données et un principe de reproductibilité de la méthode à toute portion construite du territoire, nous devons accepter l'idée que les modèles urbains à manipuler sont souvent limités à une collection d'empreintes de bâtiments et de parcelles, de lignes axiales de voirie, etc. En France, la BD Topo ${ }^{\circledR}$ de l'IGN est, en termes de couverture spatiale du territoire national, un élément de réponse satisfaisant. Ce parti-pris de minimalisme du jeu de données utilisées, nous incite à assimiler l'espace urbain, en grossière approximation, à un ensemble de "pleins » (les bâtiments) et de «vides» (l'espace interstitiel entre les bâtiments). Ces pleins et ces vides sont séparés par des enveloppes de bâtiments, façades opaques que nous estimons totalement réfléchissantes d'un point de vue acoustique. Enfin, nouvelle contrainte de travail forte, nous travaillons sur une ville bidimensionnelle, totalement sans «épaisseur» (en faisant abstraction des élévations de la couche bâtie). Cette approche «dans le plan », nous semble conforme à notre parti pris anthropocentré, dans la mesure où la perception à hauteur de piéton immergé dans l'espace ouvert urbain est principalement fonction de sa position dans le plan de ville.

Ce choix minimaliste d'une part et d'analyse morphométrique dans le plan d'autre part, présente plusieurs avantages :

- du fait même de la simplicité de formulation de l'approche, les bases de données requises ne nécessitent aucun champ attributaire (de type alphanumérique) et une géométrie simplifiée (empreinte au sol de bâtiments),

- il est plus rapide (moins de données à traiter),

- les bases de données sont plus à jour (car plus faciles à entretenir),

- un calcul d'indicateur 3D est généralement approximatif dans la mesure où les données volumiques à disposition sont toujours imprécises (nature des toits, pentes des toitures, présence de balcons...). Une approche 2D, structurellement moins exigeante en termes de qualité de données, sera de facto plus précise. L'application de cette limitation dimensionnelle à l'approximation de la diffusivité acoustique de l'espace urbain nécessite cependant une hypothèse complémentaire d'alignement des hauteurs de bâtiment. Une telle hypothèse est parfaitement recevable dans le cas du centre médiéval de Nantes, qui constitue le cas d'étude présenté ci-après, où la hauteur moyenne est de $\mathrm{R}+3$ à un

\footnotetext{
${ }^{4}$ Un isovist correspond au bassin de visibilité (sur $360^{\circ}$ ou $4 \pi$ stéradians) d'un sujet dans un environnement architectural ou urbain. C'est l'ensemble des points du plan ou de l'espace visibles depuis le point de vue. Un champ d'isovists est un ensemble d'isovists pour un environnement urbain donné.
} 
niveau près. L'hypothèse de champ diffus ${ }^{5}$ qui s'applique sur notre tissu urbain correspond donc à un modèle de rue canyon à hauteur fixe et à largeur variable.

\section{Approche par les "Visibility studies"}

Nous avons opté pour une approche qui s'inscrit dans le registre des «Visibility studies » et tire parti de la méthode à base de champs d'isovists telle que décrite dans (Benedikt, 1979).

Cette approche permet en effet de combiner une couverture systématique du territoire (approche orientée champ) avec une connaissance locale assez complète (puisque l'isovist permet, par définition, de délimiter l'espace ouvert - non construit - directement accessible depuis le point de vue aussi appelé point de génération). Ce mode d'analyse du territoire permet d'interroger la forme du déroulé de façades immédiatement environnant et ses ruptures, ainsi que les rapports plein/vide dans ce front bâti (par le biais de l'occlusivité).

\section{- Isovist}

Un isovist (Benedikt, 1979) est constitué de l'ensemble des points de l'espace qui sont visibles depuis un point donné appelé point de vue ou point de génération (voir figure 1). Dans le cas où aucune surface opaque n'arrête la portée du champ de vision, il est parfois nécessaire d'imposer un horizon artificiel pour en limiter la portée. Dans un espace plan, un isovist est une portion plane du champ de vision parallèle au sol. C'est un polygone qui présente une propriété de convexité locale au sens où le point de vue relie tout point de la forme par un segment contenu dans cette même forme.

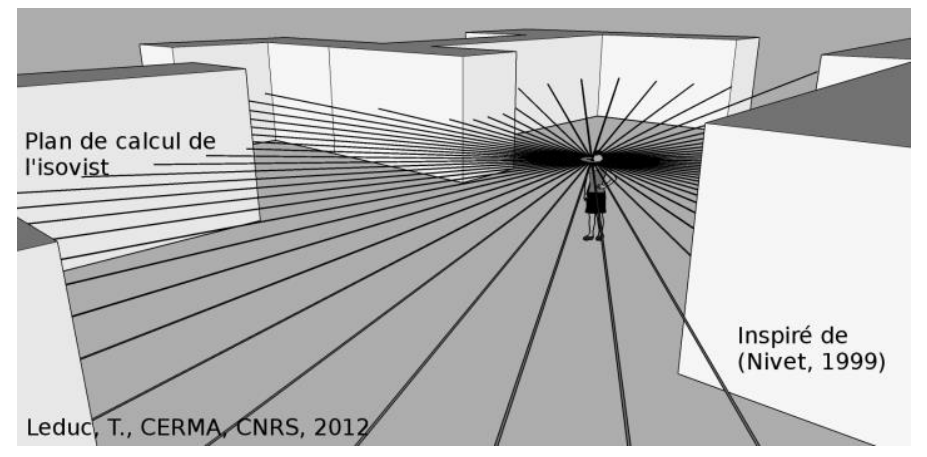

Fig. 1 : représentation symbolique de l'isovist d'après (Nivet, 1999). L'observateur est assimilé à un « capteur visuel » ayant une ouverture d'angle totale ( $4 \pi$ stéradians ou $360^{\circ}$ dans le plan de vision parallèle au sol).

\section{- Le champ d'isovists}

Pour quantifier/qualifier l'espace de façon plus systématique sur un territoire, (Benedikt, 1979) propose d'analyser le champ d'isovists. Il s'agit d'une extrapolation de la notion mathématique de champ de vecteurs qui, à tout point de l'espace euclidien, associe un vecteur. Si l'isovist décrit l'espace environnant un point donné, le champ d'isovists associe, à tout point du territoire d'étude, un isovist. Le champ d'isovists permet donc d'accéder à la propriété de description de l'espace enveloppant en tout point d'intérêt.

\section{De l'intérêt d'un indicateur informationnel pour analyser la forme environnante}

La réduction de dimension consiste à reformuler un problème exprimé dans un espace de dimension donnée dans un sous-espace de moindre dimension. En l'occurrence, nous allons restreindre l'analyse des divers isovists produits (polygones de dimension 2) à l'étude de leurs contours (géométries de dimension 1).

\footnotetext{
${ }^{5}$ L'hypothèse de champ diffus caractérise la propagation du son en enceinte close. Idéalement, le champ diffus est caractérisé par l'équirépartition du niveau sonore dans la totalité de l'espace de propagation. Le niveau d'énergie acoustique est alors indépendant de la distance à la source.
} 


\section{- Discrétisation par abscisse angulaire et fonction de distances radiales au contour}

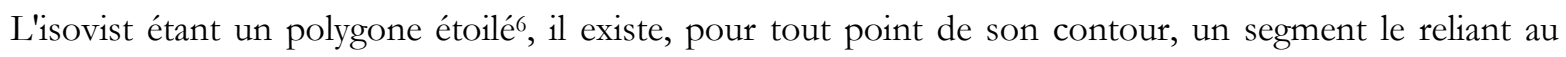
point de génération de l'isovist (segment contenu dans l'isovist). Nous pouvons donc considérer que, en utilisant une fréquence d'échantillonnage adaptée (théorème d'échantillonnage de Nyquist-Shannon), une discrétisation en abscisses angulaires ${ }^{7} \mathrm{du}$ contour constitue une bonne approximation de la forme polygonale de départ. Une telle discrétisation produit une fonction d'une variable réelle qui associe à une valeur d'angle une longueur de rayon.

\section{- L'entropie de Shannon de cette fonction de distances radiales comme un indicateur de prévisibilité/complexité du contour}

Comme rappelé dans (Salat, 2011), la complexité génère de la diversité au sein d'un tissu urbain, par exemple en maximisant les surfaces de contact, d'échange et d'interface. Pour quantifier cette complexité à l'échelle du déroulé de façades évalué en tout point de l'espace, nous avons recourt à l'entropie telle qu'introduite dans la théorie de l'information formulée par (Shannon, 1948). En effet, originellement destinée à quantifier la prévisibilité de l'information transmise au sein d'un message, les applications de cet outil ont depuis largement dépassé le strict cadre de la théorie mathématique de l'information.

Dans le contexte qui nous intéresse, la grandeur que nous représentons dans le champ scalaire est l'entropie de Shannon de la fonction de distances radiales en tout point de discrétisation. Cette grandeur est une mesure de variabilité (complexité) des longueurs de rayons du point de génération aux points du contour (issus de l'échantillonnage par abscisses angulaires). Quand cette valeur tend vers 0 , la variabilité des distances au contour décroît. Le cas limite pour lequel la valeur de l'entropie est nulle correspond au cas où le point de génération est placé au centre d'une place circulaire close.

\section{De la complexité du contour au potentiel de diffusivité acoustique}

Les travaux relatifs à la propagation du son en milieu construit ont déjà montré l'influence de la diffusion des façades sur la propagation acoustique dans une rue (Picaut et al., 2006). Le rapport entre la part d'énergie spéculaire ${ }^{8}$ et la part d'énergie diffuse ${ }^{9}$ est souvent défini comme le degré de diffusion (Heutschi, 1995) des façades. L'utilisation de ce rapport en acoustique des salles a fait l'objet d'un certain nombre de critiques (Kuttruff, 2009), tout en montrant de grandes différences sur le temps de réverbération ${ }^{10}$ et l'atténuation sonore ${ }^{11}$ dans une rue, selon que la réflexion est considérée comme totalement spéculaire ou totalement diffuse (Kang, 2000 ; Woloszyn et al., 2010a, 2010b).

Ces effets notables des lois de réflexion ${ }^{12}$ sur la propagation acoustique dans une rue peuvent être pris en compte par l'estimation de la distribution des distances entre le point de positionnement anthropocentré (point d'écoute ou point de vue) et la géométrie des façades d'un espace urbain. Ainsi, par exemple, l'asymétrie des longueurs de rayons correspond à une estimation de «dissymétrie » du

\footnotetext{
${ }^{6}$ Nous appelons polygone étoilé toute forme polygonale qui, comme écrit précédemment, présente une propriété de convexité locale (au sens où le point de vue relie tout point de la forme par un segment contenu dans cette même forme).

${ }^{7}$ Dans une telle discrétisation, l'espace angulaire est uniformément échantillonné et, à chaque valeur d'angle (équi-distribuée sur l'ensemble de l'espace angulaire), est associée la longueur du rayon correspondant.

${ }^{8}$ L'énergie spéculaire caractérise la partie réfléchie du son après avoir rencontré un plan (ou obstacle) réfléchissant, répondant à la loi de Lambert : angle d'incidence = angle de réflexion. Cette loi correspond à la loi de Braggs en optique et en photonique.

${ }^{9}$ L'énergie diffuse caractérise la partie diffusée du son après sa rencontre avec un ou plusieurs obstacles, répondant à la loi de diffusion (équirépartition énergétique dans l'espace de propagation)

${ }^{10}$ Le temps de réverbération d'un espace est la mesure la plus ancienne de caractérisation de la diffusion acoustique : c'est E. W. Sabine qui a établi la loi empirique qui définit la durée d'extinction du son en fonction du volume de l'espace de propagation et la qualité spéculaire de ses surfaces constitutives.

${ }^{11}$ L'atténuation sonore caractérise la différence de niveau entre deux points de l'espace.

${ }^{12}$ En l'occurrence : spéculaire ou diffuse. A noter qu'en acoustique, la distinction entre diffusion et diffraction n'est pas toujours claire, le terme anglais de scattering décrivant indifféremment les deux phénomènes.
} 
phénomène de diffusion sonore induisant une plus ou moins grande homogénéité du champ sonore dans l'espace environnant. Dans un registre un peu différent, la dispersion des longueurs des rayons autour d'une longueur moyenne (écart-type) permet de déterminer la dissymétrie fréquentielle de la diffusion sonore de l'espace d'étude.

La fonction de distribution des isovists permet de prédire, par exemple, une réponse acoustique en cas de mise en situation d'une source sonore. La diffusion peut être décrite comme une suite de déplacements particulaires indépendants et isotropes (à directions équiprobables), dont la longueur de déplacement répond à une distribution gaussienne de densité ${ }^{13}$ des isovists de l'espace de propagation, (le cadre bâti), dont l'entropie constitue une mesure.

Pour résumer, le calcul de l'entropie de la fonction des distances radiales des isovists permet de déterminer le temps de réverbération au point de réception (point de génération de l'isovist) par l'évaluation de la répartition des surfaces de réflexion acoustique de l'espace environnant (Woloszyn, 2000).

Un indice de potentialité de diffusion peut ainsi être évalué par cette méthode purement géométrique des isovists, en considérant la couche bâtie comme un ensemble d'arêtes (les murs de bâtiments) perturbant la propagation du son jusqu'à l'auditeur. Ces hypothèses de travail excessivement fortes nous permettent non pas de reproduire une réalité acoustique de terrain (surtout en l'absence de sources sonores) mais bien, à partir d'une évaluation systématique de la morphologie de l'espace ouvert immédiatement environnant, l'estimation d'une potentialité de diffusion des façades.

\section{Cas d'application et discussion}

Pour vérifier l'applicabilité de notre méthode, nous avons opté pour un terrain du centre historique de la ville de Nantes (France). Ce terrain, partie du quartier Bouffay, a été choisi pour ses caractéristiques morphologiques et topographiques adaptées à l'ensemble des contraintes d'application que nous avons recensées. Il s'agit effectivement d'un centre urbain dense, relativement homogène, où cohabitent des bâtiments d'élévation sensiblement constante et où le différentiel d'altitude au sol sur la totalité de l'espace est quasi nul.

Dans la portion qui nous intéresse plus précisément, zone de $0,5 \mathrm{~km}^{2}$ comprise entre le cours des cinquante otages (à l'ouest et au nord), la rue de Strasbourg (à l'est) et un tronçon de ligne de tramway $\mathrm{n}^{\circ} 1$ (au sud), nous avons opté pour une discrétisation spatiale de résolution métrique. Les 500000 points ainsi obtenus ont ensuite, pour ceux situés en extérieur du moins (hors empreintes bâties), été assimilés à des points de génération d'isovists.

Le gradient de couleurs du champ scalaire présenté en figure 2 correspond à une estimation de valeurs d'entropie de la distribution de distances radiales de l'isovist en chacun des points (extérieurs) issus de la discrétisation de la zone d'étude du quartier Bouffay. Cette représentation cartographique, outre le fait qu'elle fournisse une évaluation systématique (de résolution métrique) de l'environnement ouvert « direct» en chaque point, présente l'intérêt d'offrir une lecture thématique synthétique.

\footnotetext{
${ }^{13}$ La distribution gaussienne de densité s'applique en acoustique tant dans le domaine fréquentiel que temporel. Appliquée aux Isovists, elle caractérise une distribution de longueurs de vue en tout point de l'espace de scrutation.
} 


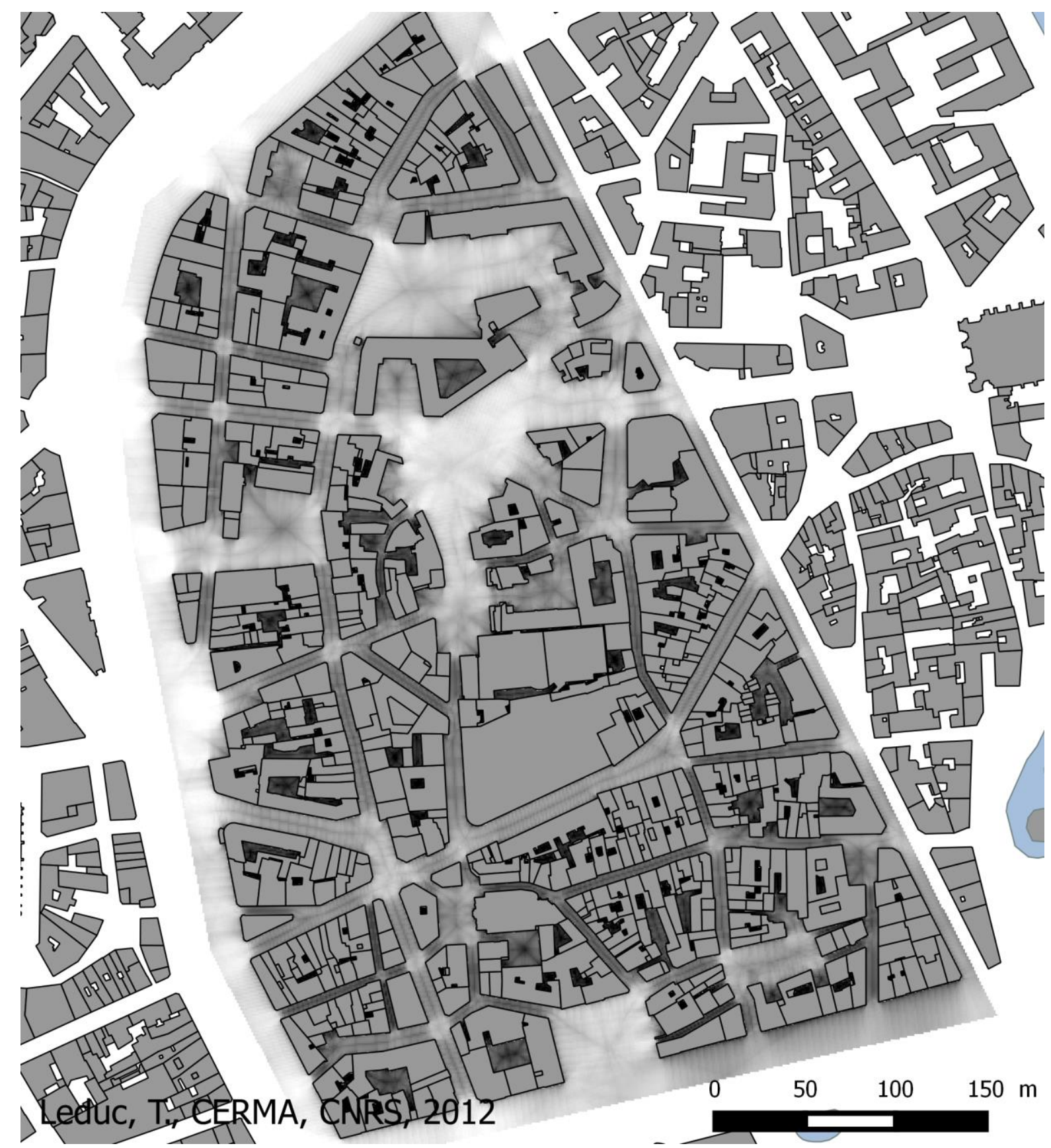

Fig. 2 : Représentation cartographique d'un champ scalaire de valeurs d'entropie de la distribution de distances radiales de l'isovist en tout point de discrétisation de l'espace ouvert du quartier du Bouffay, Nantes, France. Plus la couleur du pixel se fonce et plus l'entropie du point de vue correspondant tend vers 0.

Comme nous pouvons le constater à la lecture de cette carte, l'entropie des rues canyons de la zone tend vers 0 . Celle des places et grands boulevards se rapproche a contrario de 1 (complexité minimale du déroulé de façades environnant le récepteur). Par ailleurs, le minimum de l'entropie (valeurs proches de 0) est obtenu au centre des rues canyon du fait même d'une plus grande symétrie de distribution des rayons de visibilité. Ce sont les endroits où l'on constate une symétrie frontale et latérale des parcours de rayons sonores.

De fait, nous pouvons en conclure que les points «chauds» de la carte (pixels foncés) traduisent une forte réverbération du son dans la maille urbaine alors que les points les plus « froids» (pixels clairs) se 
rapprochent de l'hypothèse acoustique du champ libre ${ }^{14}$ (diffusion acoustique nulle). Ces faits sont corroborés par une campagne de mesure des temps de réverbération des rues du quartier Bouffay (Woloszyn, 1997).

\section{Conclusion}

Dans ce chapitre, nous nous sommes interrogés sur la possibilité, pour un indicateur morphologique 2D, de « révéler » des potentialités liées à la diffusivité acoustique en milieu urbain dense. Pour ce faire, nous avons adopté une inversion d'approche complète reposant sur la perception d'ambiances en tout point de l'espace ouvert urbain. Une telle potentialité (de diffusion) permet, par exemple, de prédire la réponse acoustique d'une source sonore, mais surtout, l'impression spatiale ressentie lors de l'audition du son.

Parce que l'approche égocentrée offre l'avantage de combiner le point de vue local (tangentiel, immergé, in situ) avec un rendu global (zénithal), nous pouvons considérer qu'elle constitue une méthode complémentaire de la technique classique de production de cartographies sonores (qualifiée précédemment d'allocentrée). L'intégrer lors du processus de production de telles cartes permettrait un réel enrichissement sémantique.

\section{Bibliographie}

Benedikt, M. L. (1979). To take hold of space: isovists and isovist fields. Environment and Planning B: Planning and Design, 6(1), 47-65.

Heutschi, K. (1995). A simple method to evaluate the increase of traffic noise emission level due to buildings, for a long straight street. Applied Acoustics, 44(3), 259-274. doi:10.1016/0003-682X(94)00027-S

Kang, J. (2000). Sound propagation in street canyons: comparison between diffusely and geometrically reflecting boundaries. The Journal of the Acoustical Society of America, 107(3), 1394-404. Retrieved from http://www.ncbi.nlm.nih.gov/pubmed/10738794

Kuttruff, H. (2009). Room acoustics (5th ed., p. 392). Taylor \& Francis Ltd.

Nivet, M.-L. (1999). De Visu: un logiciel pour la prise en compte de l'accessibilité visuelle dans le projet architectural, urbain ou paysager. Université de Nantes, ED SPI (ED 82), laboratoire CERMA, école nationale supérieure d'architecture de Nantes, France.

Picaut, J., Schmich, I., Defrance, J., Woloszyn, P., Barlet, A., \& Chartier, F. (2006). Effects of diffuse reflection by building façades on the sound propagation and soundscapes in urban areas. Euronoise 2006 Advanced solutions for noise control - The $6^{\text {th }}$ European Conference on Noise Control. Tampere, Finland.

Salat, S. (2011). Les villes et les formes: Sur l'urbanisme durable (p. 544). Hermann.

Shannon, C. E. (1948). A mathematical theory of communication. The Bell System Technical Journal, 27(1), 379-423. Retrieved from http://www.ncbi.nlm.nih.gov/pubmed/9230594

\footnotetext{
${ }^{14}$ L'hypothèse de champ libre est l'exacte opposée de l'hypothèse de champ diffus. La décroissance de l'énergie sonore est une fonction géométrique de la distance au point d'émission: le son émis décroît alors de $6 \mathrm{~dB}$ par doublement de la distance à la source.
} 
Woloszyn, P., Raymond, F., \& Picaut, J. (1997). Morphological parametering of a diffusive acoustic model for complex urban areas. Wesprai'97 $6^{\text {th }}$ Western Pacific regional acoustic conference (pp. 231-236). Hong-Kong: Hong Kong Institute of Acoustics.

Woloszyn, P. (2000). Squaring the circle: diffusion volume and acoustic behavior of a Sierpinski structure. In M. M. Novak (Ed.), Paradigms of Complexity: Fractals and Structures in the Sciences (pp. 298-300). World Scientific Publishing Company.

Woloszyn, P., \& Leduc, T. (2010a). Urban Soundscape Informational Quantization: Validation Using a Comparative Approach. Journal of Service Science and Management, 3(4), 429-439. doi:10.4236/jssm.2010.34049

Woloszyn, P., Leduc, T., \& Joanne, P. (2010b). Towards a sound diffusion characterization in the urban environment - from isovist tool to acoustic scattering indicator. IOA/ABAV Joint meeting on noise in the built environment. Gent, Belgium. 